\title{
Welfare Analysis of a Market Model with External Increasing Returns and Differentiated Commodities
}

\author{
Takashi Suzuki \\ Meiji-Gakuin University, Tokyo, Japan \\ Email: takashisuz@jcom.home.ne.jp
}

How to cite this paper: Suzuki, T. (2017) Welfare Analysis of a Market Model with External Increasing Returns and Differentiated Commodities. Theoretical Economics Letters, 7, 63-78.

http://dx.doi.org/10.4236/tel.2017.71007

Received: November 29, 2016

Accepted: January 13, 2017

Published: January 17, 2017

Copyright $\odot 2017$ by author and Scientific Research Publishing Inc. This work is licensed under the Creative Commons Attribution International License (CC BY 4.0).

http://creativecommons.org/licenses/by/4.0/

\begin{abstract}
Markets with increasing returns resulting from the positive external effects of production (hereafter external increasing returns) have been discussed from both positive and normative points of view. We present a complete general equilibrium model in which external increasing returns prevail within industries to produce differentiated commodities which are represented by real valued functions. Firms in an industry produce the commodity characteristics which are values of each commodity function. This formulation of differentiated commodities causes the equilibrium to be characterized as the solution of a variational problem. In order to answer this mathematically formidable question, we utilize the delta function of Dirac for the calculus of variations to be done in an elementary way. To our knowledge, this is the first time the delta function appears in economic analysis.
\end{abstract}

\section{Keywords}

External Increasing Returns, Division of Labor,

Differentiated Commodities, Delta Function

\section{Introduction}

In this paper, we analyze a market in which there are increasing returns resulting from a positive external effect of production. We present this idea formally, in a mathematically rigorous manner, in the following section. By increasing returns, we mean the increase in each firm's productivity resulting from the investments of all firms in the market. The theory of increasing returns in this sense was initiated by Alfred Marshall [1] with the following rather mysterious descriptions.

We may divide the economies arising from an increase in the scale of production of any kind of goods, into two classes-firstly, those dependent on the general 
development of the industry; and secondly, those dependent on the resources of the individual houses of business engaged in it, on their organization and the efficiency of their management. We may call the former external economies, and the latter internal economies (op.cit., Ch. IX, p. 221).

This ambiguous "definition" immediately invited furious debate about whether these increasing returns were compatible with the competitive equilibrium [2]-[7]. These discussions further degenerated into confusion. Indeed Chipman [8] reported as follows ${ }^{1}$.

[The compatibility of increasing returns with perfectly competitive equilibrium] was once a lively subject of debate. The debate appears to have petered out in the 1930's, with nobody the apparent winner. That this was the outcome seems evident from later writings of some of the participants. Thus, Sir Dennis Robertson [9] presented in 1957 an account that was substantially unaltered from his contribution to the 1930 Symposium on Increasing Returns, supporting the compatibility of increasing returns with perfect competition. On the other hand, Sir Roy Harrod [10] in 1967 was able to state flatly, without any qualification as to whether economies were internal or external, that "Increasing returns can, of course only occur if competition is less than perfect". In the contemporary international trade literature, some authors maintain that perfect competition can prevail under conditions of increasing returns, provided the economies of scale are external to individual firms; whereas others deny the compatibility of economies of scale with perfect competition under any circumstances, and with equal confidence ... (op.cit., pp. 347-9).

After the concept of "externalities" in the modern sense was established by Edgeworth [11], the debates were finally terminated by Chipman (op.cit.) who provided a clear definition of external increasing returns, and showed, using the example of a single-consumer economy with Cobb-Douglas utility, that external increasing returns were indeed compatible with the competitive equilibria, and even with Pareto optimality (the welfare formula).

Young (op.cit.) pointed out that this idea could date back to the division of labor in Adam Smith [12]. Young described this as follows:

The division of labor resulting in the increasing returns was limited by the extent of the market, and conversely the extent of the market is in turn enlarged by the division of labor.

Consequently, he wrote:

the division of labor depends in large part upon the division of labor, and added that

this is more than mere tautology.

Young claimed that increasing returns were the source of economic growth. This

${ }^{1}$ For details of these debates and their consequences, see also Chipman (op.cit.). 
observation was supported by Romer [13], who interpreted them as a "knowledge spillover". For example, when one firm succeeds in inventing a new technology or innovation, it will not be able to keep it secret or defend it by patent for a long period. Eventually, the discovery will be revealed to other firms, and consequently the productivity of the entire industry (and eventually the whole economy) will increase. Romer also insisted that the original idea dates back at least to Adam Smith.

The idea that increasing returns are central to the explanation of long-run growth is at least as old as Adam Smith's story of the pin factory (op.cit., p. 1004).

Why is it that both Young and Romer referred to the division of labor in Smith's pin factory? How does this relate to external increasing returns? The division of labor also increases the productivity of the firm (factory) by dividing tasks and specializing work in the production processes. However, each separate task makes no sense in and of itself. Although each part of the process is conducted by a single worker (or a group of workers), all other parts are interdependent. The division of labor then presupposes cooperative relationships between the workers in the factory. External increasing returns are then simply a generalization of reciprocity within the factory to the entire industry and/or the whole economy. As a result, external increasing returns "expand" the entire economy: they increase productivity and the range of commodities available to consumers, in addition to ensuring the efficiency of allocations. This is the source of economic growth.

In [14], the single-consumer example in Chipman (op.cit.) was generalized to a multi-consumer model which provides a consistent and reasonable model of a market with external increasing returns. The basic strategy for proving the existence of competitive equilibrium and obtaining the welfare formula for a model with several consumers is to characterize the equilibrium as the solution of a constrained social optimization problem by applying the ingenious idea of Negishi [15]. As external increasing returns arise from the externality, the Pareto optimality of the resulting equilibrium is not obvious. According to the welfare formula (see Theorem 3.3), it is indeed possible that equilibrium is optimal.

The purpose of the present paper is to incorporate differentiated commodities into the model of Suzuki (op.cit.); i.e., product diversity, in the sense of Hotelling [16], Lancaster [17], and Rosen [18], etc. The economic motivation for this mathematical generalization arises directly from the concept of external increasing returns. As already stated, (external) increasing returns are a generalization of the division of labor. The purpose of the latter is then not only to increase productivity but also to produce more varieties of commodities in society as a whole ${ }^{2}$. Therefore, it is appropriate to incorporate differentiated commodities into a market model to represent a (type of) division of labor, rather than to work with models with fixed numbers of finitely many commodities.

The optimal solution in Suzuki (op.cit.) was that of an optimization problem of a finite dimensional space. Incorporating differentiated commodities into the model, however, causes the equilibrium to be characterized as the solution of a variational

${ }^{2}$ Adam Smith (op.cit.) emphasized this. 
problem. In order to answer this mathematical question in an infinite dimensional vector space, we utilize the delta function of Dirac [19] for the calculus of variations to be done in an elementary way. To our knowledge, this is the first time the delta function appears in economic analysis.

The remainder of the paper is organized as follows. In Section 2, we present the model and define external increasing returns precisely. In Section 3, we present the theorems and proofs. Theorem 3.1 (the equilibrium formula) asserts that there exists a competitive equilibrium incorporating external increasing returns by constructing the equilibrium explicitly. This shows that the market is consistent with increasing returns. Similarly, we compute the Pareto optimal allocations (the welfare formula, Theorem 3.2). This formula enables us to compare the equilibrium and optimal allocations (Theorem 3.3). Thanks to the delta function, we do not require any mathematics beyond the level of elementary calculus to obtain the formula. In Section 4, we provide some remarks.

\section{Economy with External Increasing Returns}

\subsection{A Market Model}

There exist two categories of the commodities, the homogeneous input commodity (labor) indexed by 0 , and the differentiated consumption commodities which are determined by their characteristics indexed by $t \in T$. The index set $T$ is simply a subset of the (open) real half-line $\mathbb{R}_{++}=\{t \in \mathbb{R} \mid t>0\}$, which will be defined below. The amount of the commodity 0 is denoted by $x^{0}, z^{0}$, etc., and that of the commodity characteristic $t>0$ by $x(t), y(t)$, and so on. Mathematically, a differentiated commodity is a function defined on (a subset of) $T$. Let us call the function a commodity bundle. Then it has a continuum of coordinates, or the commodity bundle is an infinite-dimensional vector. Let $\mathcal{F}(T)$ be the set of commodity bundles.

The economic interpretation of the differentiated commodities is as follows. When two values $t \in T$ and $s \in T$ are "close" (in the usual mathematical sense), so are the commodity characteristics $t$ and $s$ (in the economic sense). In other words, the characteristics $t$ and $s$ are not exactly the same, but very similar. When each characteristic contained in two commodity bundles with the same set of the characteristics are close, they are similar, or the commodities are "differentiated". Notice that this interpretation is impossible for the usual finite-dimensional commodity bundles for which the coordinates are necessarily discrete.

Each commodity characteristic $t \in T$ is assumed to be produced from the input commodity 0 by a firm that is also indexed by $t$ for convenience (hence, there exist a continuum of firms in the economy) using the production function that embodies the increasing returns,

$$
y(t)=f_{t}\left(z_{t}^{0}, \zeta_{t}\right)
$$

$\zeta_{t}$ is a parameter that is determined by the market equilibrium. Hence, for firm $t$, the value of $\zeta_{t}$ is given by the market and not determined by the firm. The parameter $\zeta_{t}$ is assumed to be the total input, $\zeta_{t}=\int z_{s}^{0} \mathrm{~d} s$; therefore, it depends on the amount of input used by other firms in the economy (including $t$ ). That is to say, these 
increasing returns come from (positive) externalities.

We have to specify the range of the externality to extend. For simplicity, consider a finite number of (measurable ${ }^{3}$ ) subsets $\left\{T_{1} \cdots T_{n}\right\}$ of $\mathbb{R}$ such that $\sup \left\{\mid t_{j}-s_{j} \| t_{j}, s_{j} \in T_{j}\right\}<\inf \left\{\mid t_{j}-s_{j} \| t_{j} \in T_{j}, s_{k} \in T_{k}\right\}$ for all $j, k=1 \cdots n$ with $j \neq k$. We set $T=\cup_{j=1}^{n} T_{j}$ and call $T_{j}$ the industry $j$. The above condition implies that any characteristics in the same industry are closer than those of different industries.

It is important to distinguish the commodity vectors (bundles) from their characteristics. The industry $j$ produces and supplies its own commodity bundles (hence there exist $n$ distinct commodities in the economy) which are functions $x(\cdot)$ or members of an appropriate function space $\mathcal{F}\left(T_{j}\right)$ on $T_{j}$, and the characteristic $x(t)$ is their value at $t \in T_{j}$ which is not traded among the consumers. They are "intermediate goods" which constitute the commodity $j$ supplied by the industry $j$. Of course for each $t^{*} \in T$, we can consider the commodity bundle $x\left(t ; t^{*}\right)$ which contains only the characteristic $t^{*}$ defined by $x\left(t ; t^{*}\right)=1$ for $t=t^{*}$ and $x\left(t ; t^{*}\right)=0$ otherwise. But this commodity would not have any economic significance, since its market values are always 0 , as shown later on $^{4}$. Functions on $T_{j}$ can be naturally extended to $T$ setting as 0 outside $T_{j}$. Then we can write $\mathcal{F}(T)=\mathcal{F}\left(T_{1}\right) \oplus \cdots \oplus \mathcal{F}\left(T_{n}\right)$ (direct sum).

Chipman, Romer and Suzuki assumes the function $f_{t}$ to be homogeneous of degree 1 in $z^{0}$,

(Hom) $f_{t}\left(\lambda z_{t}^{0}, \zeta_{t}\right)=\lambda f_{t}\left(z_{t}^{0}, \zeta_{t}\right)$ for all $\lambda \geq 0$,

and it is monotonically increasing in $\zeta_{t}$,

(Mon) $f_{t}\left(z_{t}^{0}, \zeta_{t}\right) \leq f_{t}\left(z_{t}^{0}, \zeta_{t}^{\prime}\right)$ whenever $\zeta_{t} \leq \zeta_{t}^{\prime}$.

Then, by these two conditions, the final output $y(t)$ is produced under "increasing returns to scale"; namely,

$$
f_{t}\left(\lambda z_{t}^{0}, \lambda \zeta_{t}\right)=\lambda f_{t}\left(z_{t}^{0}, \lambda \zeta_{t}\right) \geq \lambda f_{t}\left(z_{t}^{0}, \zeta_{t}\right) \text { for all } \lambda \geq 1 \text {. }
$$

As $\zeta_{t}$ is a parameter for the firm $t$, the condition (Hom) implies that firm $t$ operates subjectively under constant returns; hence, there exist no problems involved with the "internal increasing returns (convex production functions) $)^{5}$." Furthermore, since $\zeta_{t}=\int_{T_{j}} Z_{s}^{0} \mathrm{ds}$ for $t \in T_{j}$, the condition (Mon) means that the more inputs in the industry $j$, the more productive the technology of each firm becomes. This is the source of the "increasing returns for a whole industry", and we can interpret it to be a kind of reciprocity that is naturally incorporated into the markets. "Naturally incorporated into the market" means that the value of the parameter $\zeta_{t}$ is determined endogenously, not given or postulated from outside of the market.

There exists $m$ consumers indexed by $i(=1 \cdots m)$. Following Chipman (op. cit.),

${ }^{3} \mathrm{~A}$ measurable set is a set for which its (Lebesgue) measure is well defined. Hence the integral $\int_{T_{j}} x(t) \mathrm{d} t$ is also well defined for any integrable function $x(t)$.

${ }^{4}$ In order to facilitate this point, we have to treat the commodity bundles as the equivalent classes of functions modulo null sets on $T$. Then $x\left(t ; t^{*}\right)$ is equivalent with the commodity 0 , the constant 0 function.

${ }^{5}$ For the theoretical problems arising from convex (not concave!) production functions, see Scarf [20] and Oddou [21]. Arrow [22] discussed the possibility that a kind of increasing returns exists in a more specific model. 
consumer $i$ 's utility function takes the log-linear (Cobb-Douglas) form,

$$
u_{i}\left(x_{i}(t)\right)=\int_{T} \beta_{i}(s) \log x_{i}(s) \mathrm{d} s, i=1 \cdots m,
$$

where for all $t \in T, \beta_{i}(t) \geq 0$ for each $i$ and $\sum_{i=1}^{m} \beta_{i}(t)>0$, and $\int_{T} \beta_{i}(s) \mathrm{d} s=1$, $i=1 \cdots m$. Precisely speaking, this utility function is not a function but a (non-linear) functional that is a map assigning a real value to the function $x(\cdot)$.

It is well known and can be verified easily that for log-linear utility functions, all of the standard assumptions such as continuity (in an appropriate sense ${ }^{6}$ ), (strong) concavity and monotonicity (the more consumption, the higher utility) and so on are satisfied. Note that $u_{i}$ does not include commodity 0 consumption. Hence, the consumers do not demand commodity 0 ; rather, they supply it inelastically for as long as they own it. Chipman (op.cit.) observed that this assumption makes formulae very clean without losing essence of discussion. We will then follow him.

We assume that consumer $i$ is endowed with $\omega_{i}(>0)$ units of commodity 0 as an initial endowment, but does not have an initial endowment for commodity $t$. Set $\Omega=\sum_{i=1}^{m} \omega_{i}$.

The market price of commodity 0 is denoted by $w$, and the price of commodity $t$ by $p(t)$. Then we can define the competitive equilibrium of this market in the standard manner.

Definition 2.1. An $m+1$-tuple of the input and the consumption bundles $\left(\hat{z}_{t}^{0}, \hat{x}_{i}(t)\right)_{t \in T}, i=1 \cdots m$, and the prices $(\hat{w}, \hat{p}(t))$ are called the competitive equilibrium if and only if the following conditions are satisfied.

(E-1) $\hat{x}_{i}(t)$ maximizes $u_{i}(\cdot)$ subject to $\int_{T} \hat{p}(s) x_{i}(s) \mathrm{d} s \leq \hat{w} \omega_{i}, \quad i=1 \cdots m$,

(E-2) $\hat{p}(t) f_{t}\left(z, \int_{T_{j}} \hat{z}_{s}^{0} \mathrm{~d} s\right)-\hat{w} z \leq \hat{p}(t) f_{t}\left(\hat{z}_{t}^{0}, \int_{T_{j}} \hat{z}_{s}^{0} \mathrm{~d} s\right)-\hat{w} \hat{z}_{t}^{0}=0$ for all $z \geq 0$ and for all $t \in T_{j}, \quad j=1 \cdots n$,

$$
\text { (E-3) } \sum_{i=1}^{m} \hat{x}_{i}(t)=f_{t}\left(\hat{z}_{t}^{0}, \int_{T_{j}} \hat{z}_{s}^{0} \mathrm{~d} s\right) \text { for all } t \in T_{j}, \quad j=1 \cdots n \text {, and } \int_{T} \hat{z}_{s}^{0} \mathrm{~d} s=\Omega \text {. }
$$

The economic meanings of these conditions are clear and do not require detailed explanations, apart from the production externality. Condition (E-1) is the utility maximization condition (under the budget constraint) and condition (E-2) is the profit maximization condition. As the firms perceive themselves that they operate under constant returns to scale, they earn zero profit in the equilibrium. The first condition of (E-3) states that the total amount of the characteristic $t$ which is consumed is equal to what is produced. Recall that the second variable (parameter) of the production function is the total input used within each industry $T_{j}$. These inputs sum to the total input of the whole economy $\sum_{j=1}^{n} \int_{T_{j}} z_{S}^{0} \mathrm{~d} s=\int_{T} z_{S}^{0} \mathrm{~d} s$, and the latter is equal to the total endowment of the economy (the resource is not wasted). This is consistent with the first condition of (E-3), which states that supply equals demand in the input commodity market.

An important remark on the concept of prices in this economy should be in order.

${ }^{6} \mathrm{We}$ have to specify a topology on the space of functions $x(t)$ to make the meaning of "continuity" mathematically rigorous. We set aside of this problem here, because we do not discuss topologies in the present paper. For a precise discussion, see [23]. 
Strictly speaking, given the price function $p(t)$ as in Definition 2.1, the market price is a (non-negative) linear functional $\boldsymbol{p}$ on $\mathcal{F}(T)$ defined by $\boldsymbol{p} x(t)=\int_{T} p(s) x(s) \mathrm{d} s$ for every $x(t) \in \mathcal{F}(T)$. Since the characteristic $t$ is not traded by consumers, each value $p(t)$ of the functional is not directly observable for them, or $p(t)$ is the hedonic price in the sense of Rosen (op.cit). To see this, suppose that the price functional is strictly positive, $p(t)>0$ for all $t \in T$. If $p\left(t^{*}\right)$ is observable in the market for some $t^{*} \in T$, it must be the market value of the commodity $x\left(t ; t^{*}\right)$ defined above. However, we have $\boldsymbol{p} x\left(t ; t^{*}\right)=\int_{T} p(s) x\left(s ; t^{*}\right) \mathrm{d} s=0 \neq p\left(t^{*}\right)(>0)$. This is exactly what we stated earlier. Here the industry $j$ is acting as a fictitious producer "selling" the consumption bundle $x(\cdot) \in \mathcal{F}\left(T_{j}\right)$ as a final output at the price $\boldsymbol{p}$ and "buying" infinitely many characteristics $x(t) \in \mathbb{R}$ as inputs at the prices $p(t)$.

Our formulation of the differentiated commodities forces us to present the set of firms producing commodity characteristics as a continuum $T$. On the contrary, the set of consumers is a finite set $\{1 \cdots m\}$. Therefore one might say that the latter should also be a continuum. We answer this conceptual question by noticing that our model can include a continuum of consumers, following the well known idea (e.g., [24]). Let $I=[0,1]$ be the set of consumers which is the unit interval. Define the sets

$I_{i}(i=1 \cdots m)$ by $I_{i}=\left\{a \in I \mid \frac{i-1}{m} \leq a<\frac{i}{m}\right\}, \quad i=1 \cdots m-1, \quad I_{m}=\left\{a \in I \mid \frac{m-1}{m} \leq a \leq 1\right\}$. We define the consumption sector of the economy as a "simple map" $a \mapsto\left(u_{i}\left(x_{i}(t), \omega_{i}\right)\right)$ for $a \in I_{i} \quad(i=1 \cdots m)$, here we have defined the allocations $x_{a}(t)$ for $a \in I$ is also as a simple map; $x_{a}(t)=x_{i}(t)$ for $a \in I_{i}$. Then this model presenting a continuum economy with " $m$ types" of consumers formally coincides with the finite economy of the present paper.

The next example, although economically simple, illustrates the mathematical structure of the problem and a technical devise called the delta function for elucidating it will be introduced.

\subsection{A Simple Example}

There exists one consumer $m=1$ with the utility function

$$
u(x(t))=\int_{T} \beta(s) \log x(s) \mathrm{d} s
$$

such that $\beta(t) \geq 0$ for all $t \in T$ and $\int_{T} \beta(s) \mathrm{d} s=1$. The consumer's initial endowment $\omega(>0)$ is the total endowment (resources) of the economy. Suppose for simplicity that $n=1$ so that $T_{1}=T$ (there exists only one industry in the economy).

In order to elucidate the functional calculus in an elementary way, the celebrated Dirac's delta function is used. For any $t \in T$, the "function" $\delta(t)$ on $\mathbb{R}$ is defined as

$$
\delta(t)=\left\{\begin{array}{ccc}
0 & \text { for } & t \neq 0 \\
+\infty & \text { for } & t=0
\end{array}\right.
$$

and assumed to satisfy $\int_{\mathbb{R}} \delta(s) \mathrm{d} s=1$. From this and the definition, we obtain that $\int_{T} g(s) \delta(t-s) \mathrm{d} s=g(t)$ for any function $g(s)$ (Dirac (op.cit., p.59)).

Let $f(x)$ be a differentiable function. We define 


$$
\frac{\mathrm{d} f(x(s))}{\mathrm{d} x(t)} \equiv \lim _{h \rightarrow 0} \frac{f(x(s)+h \delta(t-s))-f(x(s))}{h}=f^{\prime}(x(s)) \delta(t-s) .
$$

This is a fundamental mathematical formula that will be used throughout the paper. Then we can differentiate $\int_{T} f(x(s))$ ds with respect to $x(t)$,

$$
\frac{\mathrm{d}}{\mathrm{d} x(t)} \int_{T} f(x(s)) \mathrm{d} s=\int_{T} \frac{\mathrm{d} f(x(s))}{\mathrm{d} x(t)} \mathrm{d} s=\int_{T} f^{\prime}(x(s)) \delta(t-s) \mathrm{d} s=f^{\prime}(x(t)) .
$$

The consumer maximizes the utility function (functional) (2.3) subject to the budget constraint $\int_{T} p(s) x(s) \mathrm{d} s=w \omega$. Mathematically speaking, this requires us to solve a constrained variational problem. To this end, we differentiate the constrained Lagrangian with the multiplier $\mu$

$$
\mathcal{L}=\int_{T} \beta(s) \log x(s) \mathrm{d} s+\mu\left(w \omega-\int_{T} p(s) x(s) \mathrm{d} s\right)
$$

in $x(t)$ and obtain the first-order condition (FOC)

$$
\frac{\mathrm{d} \mathcal{L}}{\mathrm{d} x(t)}=\frac{\beta(t)}{x(t)}-\mu p(t)=0 \text {. }
$$

It follows from the FOC that $\int_{T} \beta(s) \mathrm{d} s-\mu \int_{T} p(s) x(s) \mathrm{d} s=0$; hence, $\mu=1 / w \omega$. Therefore, the demand function for $x(t)$ is given by $x(t)=(\beta(t) \omega)(w / p(t))$. The equilibrium relative price $\hat{w} / \hat{p}(t)$ can be obtained from the firm's profit condition $\hat{p}(t) f_{t}(1, \omega)=\hat{w}$.

The above calculation of equilibrium for the one-consumer economy is straightforward. Our economic problem is to compute the competitive equilibrium for the multiconsumer economy. This will be achieved in the next section, using the method of Negishi (op.cit.).

\section{Existence and Efficiency of the Equilibrium}

\subsection{The Competitive Equilibrium}

Given $\zeta=\left(\zeta_{1} \cdots \zeta_{n}\right) \in[0, \Omega]^{n}$, consider the constrained variational problem

$$
\begin{gathered}
P(\zeta) \text { :Maximize } \sum_{i=1}^{m} \alpha_{i} u_{i}\left(x_{i}(t)\right) \text { subject to } \\
\sum_{i=1}^{m} x_{i}(t) \leq f_{t}\left(z_{t}^{0}, \zeta_{j}\right), t \in T_{j}, j=1 \cdots n, \text { and } \int_{T} z_{s}^{0} \mathrm{~d} s \leq \Omega,
\end{gathered}
$$

where $\alpha_{1} \cdots \alpha_{m}$ are welfare weights of consumers satisfying $\alpha_{i} \geq 0$ and we normalize $\sum_{i=1}^{m} \alpha_{i}=1$. Let $\Delta=\left\{\left(\gamma_{i}\right) \in \mathbb{R}_{+}^{m} \mid \sum_{i=1}^{m} \gamma_{i}=1\right\}$ be the unit simplex. Then $\alpha \in \Delta$. Notice that the normalization of the welfare weights is arbitrary, but each normalization determines a price normalization, as we will see later on. The solution of this problem is a saddle point of the constrained Lagrangian

$$
\begin{aligned}
\mathcal{L}_{\alpha, \zeta}\left(\left(x_{i}(t)\right), z_{t}^{0}, p(t), w\right)= & \sum_{i=1}^{m} \alpha_{i} \int_{T} \beta_{i}(s) \log x_{i}(s) \mathrm{d} s \\
& +\sum_{j=1}^{n} \int_{T_{j}} p(s)\left(f_{s}\left(z_{s}^{0}, \zeta_{j}\right)-\sum_{i=1}^{m} x_{i}(s)\right) \mathrm{d} s \\
& +w\left(\Omega-\int_{T} z_{s}^{0} \mathrm{~d} s\right),
\end{aligned}
$$


where $p(t)$ and $w$ are the multipliers (a fortiori they will be the equilibrium prices).

The saddle point is unique by the strict concavity of the utility functional. Let the saddle point be $\left(\left(\hat{x}_{i}(t)\right), \hat{z}_{t}^{0}, \hat{p}(t), \hat{w}\right)$ which satisfies

$$
\mathcal{L}_{\alpha, \zeta}\left(\left(x_{i}(t)\right), z_{t}^{0}, \hat{p}(t), \hat{w}\right) \leq \mathcal{L}_{\alpha, \zeta}\left(\left(\hat{x}_{i}(t)\right), \hat{z}_{t}^{0}, \hat{p}(t), \hat{w}\right) \leq \mathcal{L}_{\alpha, \zeta}\left(\left(\hat{x}_{i}(t)\right), \hat{z}_{t}^{0}, p(t), w\right)
$$

for every $\left(\left(x_{i}(t)\right), z_{t}^{0}, p(t), w\right)$. For each $\alpha \in \Delta$, define a map $\Phi: \Delta \times[0, \Omega]^{n} \rightarrow \Delta \times[0, \Omega]^{n}$,

$$
\Phi\left(\left(\alpha_{i}\right),\left(\zeta_{j}\right)\right)=\left(\left(\tilde{\alpha}_{i}\right),\left(\int_{T_{j}} \hat{z}_{s}^{0} \mathrm{~d} s\right)\right)
$$

where $\tilde{\alpha}=\left(\tilde{\alpha}_{i}\right)$ is defined by

$$
\tilde{\alpha}_{i}=\frac{\max \left\{0, \alpha_{i}+\hat{w} \omega_{i}-\int_{T} \hat{p}(s) \hat{x}_{i}(s) \mathrm{d} s\right\}}{\sum_{i=1}^{m} \max \left\{0, \alpha_{i}+\hat{w} \omega_{i}-\int_{T} \hat{p}(s) \hat{x}_{i}(s) \mathrm{d} s\right\}}, i=1 \cdots m .
$$

Note that $\sum_{i=1}^{m} \max \left\{0, \alpha_{i}+\hat{w} \omega_{i}-\int_{T} \hat{p}(s) \hat{x}_{i}(s) \mathrm{d} s\right\} \neq 0$, since otherwise we would have $\alpha_{i}+\hat{w} \omega_{i}-\int_{T} \hat{p}(s) \omega_{i}(s) \mathrm{d} s \leq 0$ for all $i=1 \cdots m$. Then it follows from (3.2) that $1=\sum_{i=1}^{m} \alpha_{i} \leq \int_{T} \hat{p}(s) \sum_{i=1}^{m} \hat{x}_{i}(s) \mathrm{d} s-\hat{w} \sum_{i=1}^{m} \omega_{i} \leq 0$, a contradiction. Hence $\tilde{\alpha}=\left(\tilde{\alpha}_{i}\right) \in \Delta$ is well defined.

Suppose that there exists a fixed point ${ }^{7}\left(\left(\hat{\alpha}_{i}\right),\left(\hat{\zeta}_{j}\right)\right)$ of the map $\Phi$,

$$
\Phi\left(\left(\hat{\alpha}_{i}\right),\left(\hat{\zeta}_{j}\right)\right)=\left(\left(\hat{\alpha}_{i}\right),\left(\int_{T_{j}} \hat{z}_{s}^{0} \mathrm{~d} s\right)\right) .
$$

We will show that the saddle point $\left(\left(\hat{x}_{i}(t)\right), \hat{z}_{t}^{0}, \hat{p}(t), \hat{w}\right)$ associated with the fixed point is a competitive equilibrium. We first note that the equilibrium price vector $(\hat{p}(t), \hat{w})$ is strictly positive. Suppose $\hat{p}\left(t_{0}\right)=0$ for some $t_{0}$. Then defining a new allocation $\left(y_{i}(t)\right)$ by $y_{i}(t)=\hat{x}_{i}(t)$ for $t \neq t_{0}, \quad y_{i}\left(t_{0}\right)=\hat{x}_{i}\left(t_{0}\right)+\epsilon$ for some $\epsilon>0$, $\mathcal{L}_{\alpha, \zeta}\left(\left(y_{i}(t)\right), z_{t}^{0}, \hat{p}(t), \hat{w}\right)>\mathcal{L}_{\alpha, \zeta}\left(\left(\hat{x}_{i}(t)\right), \hat{z}_{t}^{0}, \hat{p}(t), \hat{w}\right)$, contradicting the first inequality of (3.2). $\hat{w}>0$ can be proved similarly. From the saddle point property (3.2) together with the strict positivity of prices, we conclude that the constraints of the problem $P(\zeta)$ hold with the exact equalities,

$$
\sum_{i=1}^{m} \hat{x}_{i}(t)=f_{t}\left(\hat{z}_{t}^{0}, \int_{T_{j}} \hat{z}_{s}^{0} \mathrm{~d} s\right), t \in T_{j}, j=1 \cdots n \text {, and } \int_{T} \hat{z}_{s}^{0} \mathrm{~d} s=\Omega,
$$

or the equilibrium condition (E-3) of Definition 2.1 is met.

Next we claim $\hat{\alpha}_{i}>0$ for all $i=1 \cdots m$. If not, $\hat{\alpha}_{l}=0$ for some $l$. It follows from (3.4) that $0<\hat{w} \omega_{i} \leq \int_{T} \hat{p}(s) \hat{x}_{i}(s) \mathrm{d} s$, hence $\hat{x}_{i}(t)>0$. Since $\hat{\alpha} \in \Delta, \hat{\alpha}_{h}>0$ for some $h$. Define a new allocation $\left(\tilde{y}_{i}(t)\right)$ by $\tilde{y}_{l}(t)=0$,

$\tilde{y}_{h}(t)=\hat{x}_{h}(t)+\hat{x}_{l}(t)$ and $\tilde{y}_{k}(t)=\hat{x}_{k}(t)$ for $k \neq l, h$. Then

$f_{s}\left(\hat{z}_{s}^{0}, \int_{T} \hat{z}_{s}^{0} \mathrm{~d} s\right)=\sum_{i=1}^{m} \tilde{y}_{i}(s)$ and

$$
\begin{aligned}
\mathcal{L}_{\hat{\alpha}, \hat{\xi}}\left(\left(\hat{x}_{i}(t)\right), \hat{z}_{t}^{0}, \hat{p}(t), \hat{w}\right) & =\sum_{i=1}^{m} \hat{\alpha}_{i} u_{i}\left(\hat{x}_{i}(t)\right)<\sum_{i=1}^{m} \hat{\alpha}_{i} u_{i}\left(\tilde{y}_{i}(t)\right) \\
& =\mathcal{L}_{\hat{\alpha}, \hat{\xi}}\left(\left(\tilde{y}_{i}((t)), \hat{z}_{t}^{0}, \hat{p}(t), \hat{w}\right)\right),
\end{aligned}
$$

${ }^{7}$ We don't have to prove the existence, since we will compute it explicitly in the following; see Theorem 3.1. 
contradicting the first inequality of (3.2).

Setting $\left(\alpha_{i}\right)=\left(\hat{\alpha}_{i}\right)$ and $\left(\zeta_{j}\right)=\left(\int_{T_{j}} \hat{z}_{t}^{0} \mathrm{~d} t\right)$ in the FOC's for $\mathcal{L}_{\alpha, \zeta}$, we obtain

$$
\begin{gathered}
\frac{\partial \mathcal{L}_{\hat{\alpha}, \hat{\zeta}}}{\partial x_{i}(t)}=\hat{\alpha}_{i} \beta_{i}(t) / \hat{x}_{i}(t)-\hat{p}(t)=0, i=1 \cdots m, t \in T, \\
\frac{\partial \mathcal{L}_{\hat{\alpha}, \hat{\zeta}}}{\partial z_{t}^{0}}=\hat{p}(t) f_{t}\left(\hat{z}_{t}^{0}, \int_{T_{j}} \hat{z}_{s}^{0} \mathrm{~d} s\right)-\hat{w} \hat{z}_{t}^{0}=0, t \in T_{j}, j=1 \cdots n .
\end{gathered}
$$

Since $\hat{\alpha}$ is a fixed point, it follows from the definition (3.4) that

$$
\hat{\alpha}_{i}=\frac{\hat{\alpha}_{i}+\left(\hat{w} \omega_{i}-\int_{T} \hat{p}(s) \hat{x}_{i}(s) \mathrm{d} s\right)}{1+\sum_{i=1}^{m}\left(\hat{w} \omega_{i}-\int_{T} \hat{p}(s) \hat{x}_{i}(s) \mathrm{d} s\right)}, i=1 \cdots m,
$$

and from this we obtain that

$$
\hat{w} \omega_{i}-\int_{T} \hat{p}(s) \hat{x}_{i}(s) \mathrm{d} s=\hat{\alpha}_{i} \sum_{i=1}^{m}\left(\hat{w} \omega_{i}-\int_{T} \hat{p}(s) \hat{x}_{i}(s) \mathrm{d} s\right), i=1 \cdots m .
$$

The conditions $\hat{\alpha}_{i}>0,(3.6)$ and (E-3) imply that the right-hand side of equation (3.8) is equal to 0 ; hence, the budget constraint $\int_{T} \hat{p}(s) \hat{x}_{i}(s) \mathrm{d} s=\hat{w} \omega_{i}$ follows. Let $x_{i}(t)$ be such that $\int_{T} \hat{p}(s) x_{i}(s) \mathrm{d} s \leq \hat{w} \omega_{i}$. Setting $x_{h}(t)=\hat{x}_{h}(t)$ for $h \neq i$, we obtain from the first inequality of (3.2)

$$
\hat{\alpha}_{i} u_{i}\left(x_{i}(s)\right)-\int_{T} \hat{p}(s) \hat{x}_{i}(s) \mathrm{d} s \leq \hat{\alpha}_{i} u_{i}\left(\hat{x}_{i}(s)\right)-\int_{T} \hat{p}(s) \hat{x}_{i}(s) \mathrm{d} s
$$

then

$$
\hat{\alpha}_{i} u_{i}\left(x_{i}(s)\right) \leq \hat{\alpha}_{i} u_{i}\left(\hat{x}_{i}(s)\right)+\int_{T} \hat{p}(s)\left(x_{i}(s)-\hat{x}_{i}(s)\right) \mathrm{d} s \leq \hat{\alpha}_{i} \int_{T} \beta_{i}(s) \log \hat{x}_{i}(s) \mathrm{d} s,
$$

or $u_{i}\left(x_{i}(s)\right) \leq u_{i}\left(\hat{x}_{i}(s)\right)$, hence the equilibrium condition (E-1) is met. Similarly the equilibrium condition (E-2) follows from (3.2) and (3.6).

Integrating (3.5) and using the budget constraint, we have

$$
\hat{\alpha}_{i}=\hat{w} \omega_{i}, i=1 \cdots m \text {. }
$$

This is the celebrated Negishi condition (op.cit., p. 97); the welfare weight of a consumer is the inverse of his/her marginal utility of income, which is the Lagrangian multiplier of the consumer's maximization problem (see the example of the last section). As $\sum_{i=1}^{m} \hat{\alpha}_{i}=1$, the equilibrium price has been normalized as

$$
\hat{w}=\Omega^{-1} \text {. }
$$

Summing (3.5) over $i$ with help of (3.6), (3.9) and (E-3), we obtain

$$
\begin{gathered}
\hat{w} \sum_{i=1}^{m} \beta_{i}(t) \omega_{i}=\hat{p}(t) \sum_{i=1}^{m} \hat{x}_{i}(t)=\hat{p}(t) f_{t}\left(\hat{z}_{t}^{0}, \int_{T_{j}} \hat{z}_{s}^{0} \mathrm{~d} s\right)=\hat{w} \hat{z}_{t}^{0}, \\
\text { hence } \hat{z}_{t}^{0}=\sum_{i=1}^{m} \beta_{i}(t) \omega_{i}, t \in T .
\end{gathered}
$$

For convenience, we set $\phi_{j}\left(\hat{z}_{t}^{0}\right)=f_{t}\left(\hat{z}_{t}^{0}, \int_{T_{j}} \hat{z}_{s}^{0} \mathrm{~d} s\right), t \in T_{j}$. Then we have

$$
\hat{p}(t)=\hat{z}_{t}^{0} \hat{w} / \phi_{j}\left(\hat{z}_{t}^{0}\right)=\hat{z}_{t}^{0} / \Omega \phi_{j}\left(\hat{z}_{t}^{0}\right), t \in T_{j}, j=1 \cdots n,
$$




$$
\hat{x}_{i}(t)=\left(\frac{\beta_{i}(t) \omega_{i}}{\sum_{i=1}^{m} \beta_{i}(t) \omega_{i}}\right) \phi_{j}\left(\hat{z}_{t}^{0}\right), t \in T_{j}, j=1 \cdots n, i=1 \cdots m .
$$

Therefore, we have proved

Theorem 3.1 (Equilibrium Formula) The competitive equilibrium $\left(\hat{z}_{t}^{0}, \hat{x}_{i}(t), \hat{w}, \hat{p}(t)\right)$ uniquely exists and is given by (3.10), (3.11), (3.12) and (3.13).

\subsection{The Welfare Formula}

In this section, we consider the social optimization problem

$$
\begin{gathered}
P \text { :Maximize } \sum_{i=1}^{m} \alpha_{i} \int_{T} \beta_{i}(s) \log x_{i}(s) \mathrm{d} s \text { subject to } \\
\sum_{i=1}^{m} x_{i}(t) \leq \phi_{j}\left(z_{t}^{0}\right), t \in T_{j}, j=1 \cdots n, \quad \text { and } \quad \int_{T} z_{s}^{0} \mathrm{~d} s \leq \Omega .
\end{gathered}
$$

As before, $\alpha_{1} \cdots \alpha_{m}$ are the welfare weights of consumers satisfying $\alpha_{i} \geq 0$ and $\sum_{i=1}^{m} \alpha_{i}=1$. The solution ${ }^{8}\left(\tilde{z}_{t}^{0},\left(\tilde{x}_{i}(t)\right)\right)$ of this problem is a Pareto optimal allocation, and it is a saddle point of the constrained Lagrangian

$$
\begin{aligned}
\mathcal{L}_{\alpha}\left(\left(x_{i}(t)\right), z_{t}^{0}\right)= & \sum_{i=1}^{m} \alpha_{i} \int_{T} \beta_{i}(s) \log x_{i}(s) \mathrm{d} s \\
& +\sum_{j=1}^{n} \int_{T_{j}} \mu(s)\left(\phi_{j}\left(z_{s}^{0}\right)-\sum_{i=1}^{m} x_{i}(s)\right) \mathrm{d} s+v\left(\Omega-\int_{T} z_{s}^{0} \mathrm{~d} s\right),
\end{aligned}
$$

where $\mu(t)$ and $v$ are the multipliers. The FOC's for $\mathcal{L}_{\alpha}$ are

$$
\begin{gathered}
\frac{\partial \mathcal{L}_{\alpha}}{\partial x_{i}(t)}=\alpha_{i} \beta_{i}(t) / \tilde{x}_{i}(t)-\mu(t)=0, i=1 \cdots m, t \in T, \\
\frac{\partial \mathcal{L}_{\alpha}}{\partial z_{t}^{0}}=\mu(t) \phi_{j}^{\prime}\left(\tilde{z}_{t}^{0}\right)-v=0, t \in T_{j}, j=1 \cdots n,
\end{gathered}
$$

where $\phi_{j}^{\prime}\left(z_{t}^{0}\right)=f_{t}\left(1, \int_{T_{j}} z_{s}^{0} \mathrm{~d} s\right)+\partial_{\zeta} f_{t}\left(z_{t}^{0}, \int_{T_{j}} z_{s}^{0} \mathrm{~d} s\right)$. The second term represents the externality effect of production.

The problem is: For which value of $\alpha_{i}$ should we compare the competitive allocation $\left(\hat{z}_{t}^{0},\left(\hat{x}_{i}(t)\right)\right)$ and the efficient allocation $\left(\tilde{z}_{t}^{0},\left(\tilde{x}_{i}(t)\right)\right)$ ? The answer is obviously given by the Negishi condition (3.9). From the normative point of view, this seems to be justifiable only when the initial endowments of the consumers are at least nearly identical. We will discuss this point further in the next section.

Setting $\alpha_{i}=\hat{w} \omega$ in (3.14) and summing over $i$, we have

$$
\hat{w} \sum_{i=1}^{m} \beta_{i}(t) \omega_{i}=\mu(t) \sum_{i=1}^{m} \tilde{x}_{i}(t)=\mu(t) \phi_{j}\left(\tilde{z}_{t}^{0}\right), t \in T_{j}, j=1 \cdots n .
$$

Substituting this into (3.15), it follows that

$$
\hat{w} \sum_{i=1}^{m} \beta_{i}(t) \omega_{i}\left(\frac{\phi_{j}^{\prime}\left(\tilde{z}_{t}^{0}\right)}{\phi_{j}\left(\tilde{z}_{t}^{0}\right)}\right)=v, t \in T_{j}, j=1 \cdots n .
$$

${ }^{8}$ Remember that the solution is unique. 
Multiplying $\quad \tilde{z}_{t}^{0}$ with (3.17), integrating over $T$ and summing over $j$, we obtain

$$
v=\hat{w} \Omega^{-1} \sum_{i=1}^{m} \sum_{j=1}^{n} \int_{T_{j}} \beta_{i}(s) \omega_{i}\left(\frac{\phi_{j}^{\prime}\left(\tilde{z}_{s}^{0}\right) \tilde{z}_{s}^{0}}{\phi_{j}\left(\tilde{z}_{s}^{0}\right)}\right) \mathrm{d} s .
$$

We define the elasticity $\epsilon_{j}\left(z_{t}^{0}\right)$ of firm $t$ in industry $j$ as

$$
\epsilon_{j}\left(z_{t}^{0}\right)=\left(\frac{\phi_{j}^{\prime}\left(z_{t}^{0}\right) z_{t}^{0}}{\phi_{j}\left(z_{t}^{0}\right)}\right) t \in T_{j}, j=1 \cdots n .
$$

Then substituting (3.18) and (3.19) into (3.17), we obtain

$$
\tilde{z}_{t}^{0}=\left(\frac{\epsilon_{j}\left(\tilde{z}_{t}^{0}\right)}{\Omega^{-1} \sum_{i=1}^{m} \sum_{j=1}^{n} \int_{T_{j}} \beta_{i}(s) \omega_{i} \epsilon_{j}\left(\tilde{z}_{s}^{0}\right) d s}\right) \sum_{i=1}^{m} \beta_{i}(t) \omega_{i}, t \in T_{j}, j=1 \cdots n .
$$

From (3.16) and (3.14), we finally obtain

$$
\tilde{x}_{i}(t)=\left(\frac{\beta_{i}(t) \omega_{i}}{\sum_{i=1}^{m} \beta_{i}(t) \omega_{i}}\right) \phi_{j}\left(\tilde{z}_{s}^{0}\right), t \in T_{j}, j=1 \cdots n .
$$

We have thus proved the following.

Theorem 3.2 (Welfare Formula) The optimal allocation $\left(\tilde{z}_{t}^{0}, \tilde{x}_{i}(t)\right)$ obeys the Formulaes (3.20) and (3.21).

From Theorems 3.1 and 3.2, we can immediately deduce the next theorem which was, for economies with finitely many homogeneous commodities, first stated by Chipman (op.cit., p.365) for the case $m=1$ with homogeneous commodities.

Theorem 3.3. Suppose that every consumer has a Cobb-Douglas utility functional and the same amount of labor as an endowment. Then, the optimal output (with the welfare weights proportional to the endowments) of the $t$-th product is greater than, equal to, or less than the competitive level of output according as the elasticity (at the optimum) $\epsilon_{j}\left(\tilde{z}_{t}^{0}\right)$ of the firm $t$ in the industry $j$ is greater than, equal to, or less than the weighted average of the elasticities of all industries,

$\Omega^{-1} \sum_{i=1}^{m} \sum_{j=1}^{n} \int_{T_{j}} \beta_{i}(s) \omega_{i} \epsilon_{j}\left(\tilde{z}_{s}^{0}\right) \mathrm{d} s$. In particular, if all firms elasticities are equal to the weighted average, the competitive equilibrium is Pareto optimal.

\section{Discussion}

\subsection{Formalism of Differentiated Commodities}

Ideas of differentiated commodities similar to that of the present paper date back at least to Hotelling (op.cit.). He considered a duopoly market model in which consumers of a commodity are distributed uniformly on a segment of the real line, and the two firms supplying this commodity are located at fixed places (their shops) on the segment. Each consumer demands one unit of the commodity and must go to a shop, thereby incurring a fixed cost of $c$ for each unit of distance moved. Therefore, the consumers will go to the nearest shop when the two firms charge the same price. Firms aim to set 
their prices to attract more customers and consequently to earn more profits. In this model, it is possible to interpret the commodity to be differentiated according to the coordinate of the real line (the location of the consumers), $t \in \mathbb{R}$.

Our formulation of commodity differentiation, however, follows Lancaster (op.cit.) and Rosen (op.cit.). According to them, consumers are assumed to obtain their utility not directly from the commodity itself, but from the commodity's characteristics exactly as in this paper. They assumed that there exist two (indexes of) characteristics and that the space of the commodity characteristics is a compact subset of $T$ of $\mathbb{R}^{2}$. In particular, in the Rosen model, every characteristic $t \in T$ has its own "price" $p(t)$, although it is not revealed in the market; only the commodities-rather than their characteristics-are traded in the market. Rosen called $p(t)$ the hedonic price (the title of his paper) of characteristic $t$.

Mas-Colell [25] and Jones [26] generalized these ideas of commodity differentiation into an abstract concept that treats the differentiated commodity as a measure (distribution) $\boldsymbol{m}$ on a compact metric space $T$. The measure $\boldsymbol{m}$ is a countably additive and real valued set function on $T$, meaning that for each set (precisely each "measurable" set) $B, \boldsymbol{m}(B)$ is a real value interpreted as an amount of the commodity $\boldsymbol{m}$, that contains a portion $B \subset T$ of the characteristics. Countable additivity means that $\boldsymbol{m}\left(\cup_{i=1}^{\infty} B_{i}\right)=\sum_{i=1}^{\infty} \boldsymbol{m}\left(B_{i}\right)$ for all countably many pairwise disjoint $B_{i}$ s.

In this definition, the commodities are set functions, not simply functions, as in our present paper. Mas-Colell justified this concept by saying "the choice problem [of the differentiated commodities model] is not typically how much of each (perhaps individually insignificant) commodity [characteristic] to buy, but which commodity to buy." We agree that the measures are conceptually more appropriate than the functions. However, there are mathematically formidable complications in the commodity space of the measures, and the paper of Mas-Colell is very difficult to digest. Jones (op.cit.) simplified Mas-Colell's proof considerably. Khan-Suzuki [27] further elaborated these works.

\subsection{Relation with Monopolistic Competition}

The competitive equilibrium presented in this paper can be interpreted as a monopolistically competitive equilibrium in the long run. For simplicity, consider the one consumer economy of the example in Section 2.2.

If the firm $t$ behaves monopolistically, it does not take the price $p(t)$ as given, but uses the true (inverse) demand function $p(t)=(\beta(t) \omega w) / x(t)$ to compute profit. Substituting this into the profit function $\pi(t)=p(t) f_{t}\left(z_{t}, \omega\right)-w z_{t}$, with $x(t)=f_{t}\left(z_{t}, \omega\right)$, yields $\pi(t)=w\left(\beta(t) \omega-z_{t}\right)$. The maximum profit is equal to 0 , rather than $w \beta(t) \omega$. This will be explained as follows. To maximize the profit, firm $t$ must set $z_{t}=0$, or leave the market, in which case profit is $\pi(t)=0$. Consequently, $x(t)=f(0, \omega)=0$. However, this situation is unstable because $p(t)=+\infty$ for $x(t)=0$, which implies that a firm supplying something earns an infinite profit. Then, the firm enters the market, and sets $z_{t}=\beta(t) \omega$ (any other values are inconsistent with profit maximization). The resulting profit is equal to 0 , and this is a monopolistically competitive equilibrium that is stable in the long run. Because 
$x(t)=f(\beta(t) \omega, \omega)=f(1, \omega) \beta(t) \omega$, the monopolistically competitive equilibrium coincides with the competitive equilibrium. This is exactly what we wanted to show.

\section{Conclusions}

In this paper, we have considered a theoretical model in which the external increasing returns increase the productivity of firms that produce products which are the characteristics of differentiated commodities. We have demonstrated that such a market model is compatible with competitive equilibrium, and even with Pareto optimality.

The external increasing returns are essentially a type of positive externality that works outside markets through the background institutions of societies. Romer's "knowledge spillover effect" is an example of a positive externality. We can also include educational systems, as workers' skills gained through education contribute significantly to a firm's productivity. These institutions organize participants' activities, integrating them such that they can contribute to the whole economy (and society). When the institutions work well, society behaves reciprocally and is well ordered. Adam Smith recognized reciprocity occurring within the division of labor in pin factories and placed these observations at the start of his book, suggesting its importance; his true motive was not to promote unrestricted laissez-faire.

Neoclassical economic theory has emphasized the efficiency of markets, realized through the price mechanism. It has been inclined to shun externalities as a cause of market failures. The presence of external increasing returns, however, supports an opposing view that highlights the significance of background institutions. We must bear in mind that economies do not simply work through market mechanisms; they must be supported by various institutions, both social and political. Unlimited laissezfaire is not appropriate, so it is essential to establish effective institutions that facilitate reciprocity in societies.

Finally, we point out some open questions that remain unanswered in regard to our model. The first issue is our restriction of utility functions in order to compute exact formulae. Obviously, proving the existence of equilibria in a general setting is the first task for pure theorists. Moreover, we may ask if we can obtain any general results concerning optimality. The welfare formulae seem to suggest that the optimal states are "on a knife edge" and are attained "by accident." If so, can this be stated more precisely? In other words, can the "size" of the optimal states in an appropriate parameter space be estimated? The second issue is the incompleteness of our formulation for the production process of differentiated commodity bundles as final outputs. As stated above, the theoretical status of industry $j$ was "fictitious," and thus a precise and complete description of it is strongly desired in future work.

\section{Acknowledgements}

I would like to thank Sonia Tu for her warm help and encouragement. Discussions with M. Ali Khan and Nobusumi Sagara are also helpful. Comments of an anonymous referee are gratefully acknowledged. Remaining errors and shortcomings are of course my own. 


\section{Supported}

This research is supported by a Grant-in-Aid for Scientific Research (No. 15K03362) from the Ministry of Education, Culture, Sports, Science and Technology, Japan.

\section{References}

[1] Marshall, A. (1890) Principles of Economics. Macmillan, London.

[2] Graham, F.D. (1923) Some Aspects of Production Further Considered. Quarterly Journal of Economics, 37, 199-227. https://doi.org/10.2307/1883929

[3] Graham, F.D. (1925) Some Fallacies in the Interpretation of Social Cost. A Reply. Quarterly Journal of Economics, 39, 324-330. https://doi.org/10.2307/1884879

[4] Knight, F. (1924) Some Fallacies in the Interpretation of Social Cost. Quarterly Journal of Economics, 38, 582-606. https://doi.org/10.2307/1884592

[5] Knight, F. (1925) On Decreasing Cost and Comparative Cost. A Rejoinder. Quarterly Journal of Economics, 39, 331-333. https://doi.org/10.2307/1884880

[6] Sraffa, P. (1926) The Laws of Return under Competitive Conditions. Economic Journal, 36, 535-550. https://doi.org/10.2307/2959866

[7] Young, A. (1928) Increasing Returns and Economic Progress. Economic Journal, 38, 527542. https://doi.org/10.2307/2224097

[8] Chipman, J.S. (1970) External Economies of Scale and Competitive Equilibrium. Quarterly Journal of Economics, 84, 347-385. https://doi.org/10.2307/1879425

[9] Robertson, D.H. (1957) Lectures on Economic Principles. Staples Press, London.

[10] Harrod, R.H. (1967) Increasing Returns. In: Kuenne, R.E., Eds., Monopolistic Competition Theory: Studies in Impact, Essays in Honor of Edward H. Chamberlin, John Wiley and Sons, Inc., New York, 63-79.

[11] Edgeworth, F.Y. (1925) Papers Relating to Political Economy I-III. Macmillan and Co Ltd., London.

[12] Smith, A. (1776/1976) The Wealth of Nations. Reprint Edited by Cannan, E., University of Chicago Press, Chicago.

[13] Romer, P. (1986) Increasing Returns and Economic Growth. Journal of Political Economy, 94, 1002-1037. https://doi.org/10.1086/261420

[14] Suzuki, T. (2009) General Equilibrium Analysis of Production and Increasing Returns. World Scientific, Singapore. https://doi.org/10.1142/6953

[15] Negishi, T. (1960) Welfare Economics and Existence of an Equilibrium for a Competitive Economy. Metroeconomica, 12, 92-97. https://doi.org/10.1111/j.1467-999X.1960.tb00275.x

[16] Hotelling, H. (1929) Stability in Competition. The Economic Journal, 39, 41-57. https://doi.org/10.2307/2224214

[17] Lancaster, K. (1975) Socially Optimal Product Differentiation. The American Economic Review, 65, 567-585.

[18] Rosen, S. (1974) Hedonic Prices and Implicit Markets: Product Differentiation in Pure Competition. Journal of Political Economy, 82, 34-55. https://doi.org/10.1086/260169

[19] Dirac, P.A.M. (1958) The Principles of Quantum Mechanics. 4th Edition, Oxford University Press, London.

[20] Scarf, H.E. (1986) Notes on the Core of a Production Economy. In: Hildenbrand, W. and Mas-Colell, A., Eds., Contributions to Mathematical Economics. In Honor of Gerard Debreu, North-Holland, Amsterdam, 401-429.

[21] Oddou, C. (1976) Theoremes d'Existence et d'Equivalence pour des Economies avec Pro- 
duction. Econometrica, 44, 265-281. https://doi.org/10.2307/1912723

[22] Arrow, K.J. (1962) The Economic Implications of Learning by Doing. Review of Economic Studies, 29, 155-173. https://doi.org/10.2307/2295952

[23] Suzuki, T. (2016) Market Equilibrium as a Constrained Optimal Solution. Forthcoming in International Journal of Applied and Experimental Mathematics.

[24] Hervés-Beloso, C. and Moreno-Gracía, E. (2008) Competitive Equilibria and the Grand Coalition. Journal of Mathematical Economics, 44, 697-706. https://doi.org/10.1016/j.jmateco.2006.11.002

[25] Mas-Colell, A. (1975) A Model of Equilibrium with Differentiated Commodities. Journal of Mathematical Economics, 2, 263-296. https://doi.org/10.1016/0304-4068(75)90028-2

[26] Jones, L. (1984) A Competitive Model of Commodity Differentiation. Econometrica, 52, 507-530. https://doi.org/10.2307/1911501

[27] Ali Khan, M. and Suzuki, T. (2016) On Differentiated and Indivisible Commodities: An Expository Re-Framing of Mas-Colell's 1975 Model. Advances in Mathematical Economics, 20, 103-128. https://doi.org/10.1007/978-981-10-0476-6_5

\section{Submit or recommend next manuscript to SCIRP and we will provide best service} for you:

Accepting pre-submission inquiries through Email, Facebook, LinkedIn, Twitter, etc. A wide selection of journals (inclusive of 9 subjects, more than 200 journals)

Providing 24-hour high-quality service

User-friendly online submission system

Fair and swift peer-review system

Efficient typesetting and proofreading procedure

Display of the result of downloads and visits, as well as the number of cited articles Maximum dissemination of your research work

Submit your manuscript at: http://papersubmission.scirp.org/

Or contact tel@scirp.org 